\title{
Bilan de la surveillance épidémiologique des travailleurs du cycle électronucléaire en France
}

\author{
E. Samson ${ }^{1}$, K. Leuraud ${ }^{1}$, E. Rage ${ }^{1}$, S. Caër-Lorho ${ }^{1}$, S. Ancelet ${ }^{1}$, E. Cléro ${ }^{1}$, S. Bouet ${ }^{1}$, S. Hoffmann ${ }^{1}$, \\ L. Fournier ${ }^{1}$, M. Belloni ${ }^{1}$, I. Jovanovic ${ }^{2}$, T. Bah ${ }^{3}$, E. Davesne ${ }^{4}$, E. Blanchardon ${ }^{4}$, \\ C. Challeton-de Vathaire ${ }^{4}$, D. Laurier ${ }^{1}$ et O. Laurent ${ }^{1, *}$ \\ ${ }^{1}$ Institut de Radioprotection et de Sûreté Nucléaire (IRSN), Laboratoire d'Épidémiologie des rayonnements ionisants (PSE-SANTE/ \\ SESANE/LEPID), Fontenay-aux-Roses, France. \\ 2 Amplexor, Montigny-le-Bretonneux, France. \\ 3 Amarexia, Paris, France. \\ ${ }^{4}$ Institut de Radioprotection et de Sûreté Nucléaire (IRSN), Laboratoire d'Évaluation de la Dose Interne (PSE-SANTE/SDOS/LEDI), \\ Fontenay-aux-Roses, France.
}

Reçu le 9 mars 2018 / Accepté le 18 juin 2018

\begin{abstract}
Résumé - À ce jour, les normes internationales de radioprotection sont essentiellement basées sur les connaissances des effets sanitaires des rayonnements ionisants issues des études de cohortes de survivants des bombardements atomiques d'Hiroshima et de Nagasaki, qui ont subi des irradiations par voie externe à forts débits de doses. Or, les expositions aux rayonnements ionisants survenant en population générale et chez certains travailleurs correspondent généralement à de faibles doses, cumulées de manière prolongée dans le temps. Ces expositions peuvent avoir lieu par irradiation externe mais aussi par contamination interne. Les études épidémiologiques chez les travailleurs du nucléaire permettent notamment de mieux caractériser les effets de ces conditions d'expositions. Elles permettent ainsi d'évaluer l'adéquation des normes de radioprotection pour protéger les travailleurs, mais également la population générale adulte, contre les risques de cancer. Par ailleurs, elles permettent d'aborder de nouveaux questionnements de recherche en radioprotection, comme les potentiels effets non cancéreux. Enfin, ces études fournissent un bilan de santé général des populations de travailleurs du nucléaire. Cet article propose une vue d'ensemble des recherches épidémiologiques menées par l'Institut de Radioprotection et de Sûreté Nucléaire (IRSN) chez les travailleurs du cycle électronucléaire en France. Il dresse également quelques perspectives de développements possibles de ces recherches.
\end{abstract}

Mots clés : épidémiologie / cohorte / exposition professionnelle / travailleurs / rayonnements ionisants

\begin{abstract}
Epidemiological follow-up of nuclear industry workers in France: an overview. Nowadays, protection standards against ionizing radiation health effects are mainly derived from the results of the epidemiological study of atomic bomb survivors in Hiroshima and Nagasaki, who were exposed externally to radiation, at high dose rate. However, exposure to ionizing radiation in the general population and in workers generally occurs as a cumulation of low doses over years (therefore, at low dose rates), and as a result of external, but also internal, exposure. Epidemiological studies in radiation workers allow studying the long-term health effects of such exposures, and are precious to assess the adequacy of current radiation protection standards against cancer. In addition, they also allow for the study of new priority research questions in radiation protection, such as potential non-cancer effects of low dose radiation. Finally, they also provide a general picture of health in populations of radiation workers, which is useful for occupational and public health expertise. This article provides an overview of epidemiological research conducted in workers of the nuclear cycle by the French Institute for Radiological Protection and Nuclear Safety (IRSN), and discusses perspectives for its future developments.
\end{abstract}

Keywords: epidemiology / cohort / occupational exposure / workers / ionising radiation

\footnotetext{
*Auteur de correspondance : olivier.laurent@irsn.fr
} 


\section{Introduction}

Les connaissances sur les effets sanitaires des rayonnements ionisants sont issues en grande partie du suivi épidémiologique des survivants japonais des bombardements atomiques d'Hiroshima et de Nagasaki (Ozasa et al., 2012; Grant et al., 2017), mais aussi d'autres études, épidémiologiques et expérimentales (UNSCEAR, 2008; McLean et al., 2017). Ces connaissances ont mené à l'élaboration par la Commission Internationale de Protection Radiologique (CIPR) d'un système de radioprotection visant notamment à prévenir les risques de cancers et d'effets héréditaires radioinduits chez les travailleurs et le public (ICRP, 2007).

L'apport de l'épidémiologie a donc été et demeure primordial pour la radioprotection (Bourguignon et al., 2017).

$\mathrm{Si}$ ces normes de radioprotection et les réglementations nationales en ayant résulté sont essentielles et ont fait la preuve de leur utilité, certains questionnements scientifiques demeurent concernant les effets des rayonnements ionisants à des doses dites faibles (typiquement, lorsque des rayonnements de faibles transferts linéiques d'énergie sont considérés, doses aux tissus ou au corps entier inférieures à 100 milliGrays) (UNSCEAR, 2015). Parmi ces questionnements, recensés et priorisés par plusieurs organismes internationaux (HLEG, 2009; ICRP, 2017; Repussard, 2017; Kreuzer et al., 2018), figurent l'éventualité de risques de pathologies non cancéreuses (notamment de pathologies du système circulatoire), les effets des expositions chroniques (par comparaison avec les expositions très brèves, à haut débit de dose, subies par les survivants d'Hiroshima et de Nagasaki), la forme de la relation dose-risque aux faibles doses, les effets des différentes natures de rayonnements (photons, particules alpha, neutrons...), les effets des contaminations internes par différents radionucléides ou encore les différences interindividuelles de radiosusceptibilité (Kreuzer et al., 2018). Les questions portant sur les effets des expositions chroniques et par contamination interne à faibles doses sont d'autant plus importantes en radioprotection que la plupart des expositions environnementales et professionnelles aux rayonnements ionisants correspondent à ces modes d'exposition (UNSCEAR, 2008; IRSN, 2017a).

Les études épidémiologiques chez les travailleurs du nucléaire présentent plusieurs intérêts majeurs. Sur le plan scientifique, elles visent à répondre à plusieurs questionnements de recherche prioritaires en radioprotection, parmi ceux évoqués ci-dessus. De par les conditions d'exposition des travailleurs et leurs modalités de suivi dosimétrique et sanitaire (en particulier, un suivi régulier par la médecine du travail), ces études sont particulièrement informatives pour étudier les effets sanitaires d'expositions chroniques à de faibles doses de rayonnements ionisants, qu'il s'agisse d'expositions externes (Leuraud et al., 2015 ; Richardson et al., 2015) ou internes par différents radionucléides (Laurier et al., 2012), comme par exemple le radon (NRC, 1999; Kreuzer et al., 2010, 2015b; Rage et al., 2018), le plutonium (Gilbert et al., 2013; Azizova et al., 2014; Gillies et al., 2017a; Grellier et al., 2017), l'uranium (Laurent et al., 2016a ; Samson et al., 2016; Grellier et al., 2017; Yiin et al., 2017) ou encore d'autres radioéléments (Boice et al., 2014 ; Grellier et al., 2017). Par ailleurs, ces études remplissent également un rôle essentiel de surveillance sanitaire, en fournissant des bilans de santé des populations de travailleurs étudiées. Ces informations sont utiles aux acteurs de la santé au travail, de la santé publique et de la radioprotection, et supportent l'expertise dans ces domaines (Laurier and Hill, 2013 ; Zerbib, 2018).

L'Institut de Protection et de Sûreté Nucléaire (IPSN), en collaboration avec la COmpagnie GEnérale des MAtières nucléaires (COGEMA), a mis en place dès 1982 la première étude épidémiologique sur les mineurs d'uranium en France. Par la suite l'IPSN, devenu IRSN en 2002, a développé le suivi épidémiologique de larges populations de travailleurs du cycle électronucléaire français, couvrant une période d'étude de 1945, pour les embauches les plus anciennes, à aujourd'hui. Cet article présente un bilan du suivi épidémiologique des travailleurs du cycle électronucléaire français réalisé à l'IRSN et dresse des perspectives de développement de ce suivi. Le présent article résume et vient compléter par certains aspects, notamment en mettant en perspective les résultats des études au niveau international, un rapport détaillé accessible en ligne (IRSN, 2017b).

\section{Méthodes}

Les études conduites par l'IRSN concernent des populations de travailleurs salariés des grandes entreprises de l'industrie nucléaire: le CEA, EDF, AREVA NC (anciennement COGEMA, et récemment devenue ORANO), et certaines de leurs filiales. Plusieurs cohortes couvrant les grandes étapes du cycle électronucléaire en France, décrites en détail ailleurs (IRSN, 2017b), ont été constituées.

La mise en place d'une étude de cohorte en milieu professionnel débute par une phase de faisabilité, comportant notamment la définition du périmètre de l'étude (critères d'inclusion) et la vérification de la disponibilité des données à recueillir. Cette phase aboutit à la rédaction d'un protocole d'étude. Sur la base de celui-ci, des accords doivent être obtenus de différentes entités. Au niveau national, les accords du Comité d'Expertise pour les Recherches, les Études et les Évaluations dans le domaine de la Santé (validant notamment la finalité de l'étude) et de la Commission Nationale de l'Informatique et des Libertés (garantissant les modalités de protection des données personnelles) sont indispensables. Au niveau des entreprises qui mettent à disposition leurs données pour réaliser les études, les accords de la direction, du service des ressources humaines, de la médecine du travail mais aussi des représentants du personnel par l'intermédiaire des Comités d'Hygiène, de Sécurité et des Conditions de Travail, sont également nécessaires.

Dans un objectif d'étude des effets sanitaires des expositions chroniques, seuls les travailleurs ayant été employés dans les entreprises en tant que statutaires pour une durée minimale de six mois ou d'un an (selon les études) sont inclus dans les cohortes. En effet, les travailleurs quittant une entreprise de manière précoce peuvent l'avoir fait pour des raisons variées, certaines étant liées à leur santé, sans pour autant que cet état de santé puisse refléter les effets des expositions professionnelles chroniques dans l'entreprise en question (Goldberg and Luce, 2001). De plus, l'expérience montre que la reconstitution de l'historique professionnel des travailleurs n'occupant pas un emploi stable est difficile à réaliser de manière exhaustive au cours du temps (passage dans de multiples entreprises, conservation et localisation des archives...) (Guerin et al., 2009).

Pour chaque travailleur inclus, plusieurs types de données sont systématiquement collectés : 
- des données administratives nominatives, indispensables au croisement de certaines informations provenant de sources différentes (détaillées ci-dessous);

- des données d'historiques de carrières, fournies par les services de ressources humaines des entreprises;

- des données d'exposition aux rayonnements ionisants ;

- le statut vital (vivant ou décédé, et le cas échéant la date de décès) obtenu auprès du Registre National d'Identification des Personnes Physiques (RNIPP) tenu par l'Institut national de la statistique et des études économiques (Insee);

- pour les personnes décédées, les causes médicales de décès, obtenues auprès du Centre d'épidémiologie sur les causes médicales de décès (CépiDC) de l'Institut national de la santé et de la recherche médicale (Inserm). Le CépiDC centralise l'ensemble des causes médicales de décès de la population française depuis 1968.

Des informations complémentaires peuvent être recueillies, en fonction de leur disponibilité, mais aussi des objectifs spécifiques de chaque étude et des moyens qui lui sont dédiés :

- des données complémentaires sur les causes de décès survenues avant 1968, et par conséquent non recensées par le CépiDC, ont pu être recueillies à partir de dossiers médicaux. Ce travail a été spécifiquement réalisé pour l'étude conduite chez les mineurs d'uranium (Laurier et al., 2004);

- des données sur d'autres expositions professionnelles que les rayonnements ionisants. Ces informations peuvent parfois être extraites de dossiers médicaux quand elles y sont mentionnées (fiches de postes et nuisances, fiches d'exposition), mais doivent généralement être reconstituées par des approches de matrices emplois-expositions. Le principe de ces matrices est d'établir pour chaque poste de travail des valeurs d'indices d'exposition (indices de probabilité, fréquence et niveaux d'expositions) à des nuisances, ces valeurs pouvant varier selon les périodes (Guseva Canu et al., 2009, 2013; Laurent et al., 2016b); - des données sur des facteurs de risque individuels des maladies étudiées, comme la consommation de tabac par exemple. Ces informations peuvent être issues des dossiers médicaux (Leuraud et al., 2011; Drubay et al., 2015; Zhivin et al., 2017) ou d'enquêtes ponctuelles (Leuraud et al., 2007; Guseva Canu et al., 2014).

Les données recueillies sont rendues anonymes après croisements, pour réaliser les analyses statistiques. Deux principaux types d'analyses sont réalisés :

- des bilans de mortalité sont réalisés dans un premier temps, en comparant la mortalité observée dans les cohortes à celle observée dans la population générale française. Pour cela, des Rapports de Mortalité Standardisés (Standardized Mortality Ratio, ou SMR) contrôlant l'influence de certains facteurs renseignés dans les statistiques de la population générale tels que l'âge, le sexe et la période calendaire sont calculés. Cette approche, classique en santé au travail, permet d'identifier si des excès de décès par certaines pathologies sont observés dans les cohortes par rapport au nombre de cas que l'on s'attendrait à y observer si elles présentaient les mêmes taux de mortalité que la population générale (Goldberg and Luce, 2001);
- un deuxième type d'analyse, réalisable sous réserve d'une bonne reconstitution des expositions professionnelles aux rayonnements ionisants, est de caractériser, et notamment de quantifier, la relation entre ces expositions et la mortalité. On parle alors d'analyse de relations « exposition-risque », «dose-risque » ou encore «dose-réponse ». Des modèles sont proposés pour décrire la variabilité des données épidémiologiques disponibles (ex : régression de Poisson, modèles de survie à risques proportionnels...) et formaliser les relations entre exposition aux rayonnements ionisants et risque d'intérêt (ex: structure linéaire sans seuil, linéaire quadratique...), en tenant compte de variables pertinentes comme l'âge, le sexe, la période de naissance, la durée d'emploi et de facteurs modifiants la relation entre exposition et risque, comme l'âge à l'exposition, le temps écoulé depuis l'exposition... (Daniels et al., 2017). Puis, ces modèles sont ajustés aux données disponibles afin d'estimer le risque de décès pour une pathologie donnée en fonction de l'exposition aux rayonnements ionisants. Par ailleurs, des méthodes statistiques sophistiquées, comme les approches hiérarchiques bayésiennes, sont développées afin de prendre en compte explicitement et simultanément des sources d'incertitude multiples et complexes, comme les erreurs de mesure sur les expositions radiologiques (Hoffmann et al., 2017), dans l'estimation des relations exposition-risque. Ces analyses fournissent des résultats d'un intérêt primordial pour répondre aux questionnements de recherche en radioprotection (Kreuzer et al., 2018).

Au sein d'une cohorte, des analyses de relation dose-risque peuvent être menées pour l'ensemble des causes de décès (Leuraud et al., 2015; Richardson et al., 2015; Gillies et al., 2017b). Si l'on souhaite approfondir l'étude d'une pathologie en particulier, par exemple le cancer du poumon (Leuraud et al., 2011; Grellier et al., 2017) ou les pathologies de l'appareil circulatoire (Drubay et al., 2015), et recueillir des données complémentaires sur les facteurs de risque de cette pathologie comme par exemple, le tabagisme (Leuraud et al., 2011), la glycémie, l'indice de masse corporelle ou l'hypertension (Drubay et al., 2015), la conduite d'une étude cas-témoins nichée dans une cohorte peut constituer une alternative efficiente. Ce design d'étude nécessite uniquement de récolter les informations complémentaires chez les personnes «cas» atteintes de la pathologie étudiée et chez un nombre limité de personnes «témoins» indemnes de la pathologie, sélectionnés aléatoirement au sein de la cohorte (Leuraud et al., 2011; Drubay et al., 2015 ; Grellier et al., 2017; Zhivin et al., 2017).

\section{Cohortes et principaux résultats}

Plusieurs études de cohortes menées à l'IRSN couvrent l'ensemble des étapes du cycle du nucléaire en France depuis sa mise en place (IRSN, 2017b).

\subsection{Cohorte française des mineurs d'uranium.}

Cette cohorte inclut 5400 mineurs d'uranium anciennement employés durant au moins un an entre 1946 et 2001 par le CEA ou la COGEMA. Dans le cadre des activités d'extraction 
de l'uranium, ces mineurs ont notamment été exposés au radon et à ses descendants radioactifs à vie courte, aux rayonnements gamma et aux poussières d'uranium (Rage et al., 2018). Dans cette cohorte, des excès de mortalité par tous types de cancers confondus, par cancer du poumon, par cancer du rein et par silicose sont observés par rapport à la population générale. L'exposition cumulée au radon est associée au risque de décès par cancer du poumon (ce qui est également observé dans d'autres cohortes à l'étranger [NRC, 1999]), par pathologies cérébrovasculaires (ce résultat étant isolé au plan international) et par silicose (Rage et al., 2018). Le cancer du rein n'est associé ni à l'exposition au radon, ni aux rayonnements gamma, ni aux poussières d'uranium (Drubay et al., 2014; Rage et al., 2018). La prise en compte de facteurs de risque de pathologies de l'appareil circulatoire (surcharge pondérale, hypertension, diabète, hypercholestérolémie, tabagisme...) a été explorée dans le cadre d'une enquête cas-témoins nichée portant spécifiquement sur ces pathologies. Si dans ce sousensemble, la relation entre exposition radiologique et pathologies cérébrovasculaires n'est plus statistiquement significative en raison d'un nombre plus limité de sujets pour lesquels toutes ces données étaient disponibles, l'analyse réalisée suggère que la prise en compte des facteurs de risque cardiovasculaires influe très peu sur les estimations de relations exposition-risque (Drubay et al., 2015).

La cohorte française des mineurs d'uranium a notamment été incluse dans une analyse conjointe internationale de cohortes similaires ayant confirmé l'association entre exposition au radon et risque de cancer pulmonaire (NRC, 1999) et dans des analyses cas-témoins nichées internationales ayant confirmé la persistance de cette association après prise en compte du tabagisme (Leuraud et al., 2011).

Des développements méthodologiques ont permis de prendre en compte la structure complexe des erreurs de mesure sur l'exposition au radon, identifiée dans cette cohorte, afin d'obtenir une estimation affinée de la relation entre cette exposition et le risque de décès par cancer du poumon (Allodji et al., 2012; Hoffmann et al., 2017). La persistance de l'association entre radon et cancer du poumon a été confirmée.

\subsection{Cohorte F-Millers}

Les travailleurs des usines de traitement du minerai d'uranium ou «millers», opérant en aval de l'extraction (mines) et en amont des étapes de conversion de l'uranium, présentent un profil d'exposition particulier marqué par de plus faibles expositions au radon que les mineurs mais des expositions non négligeables aux poussières d'uranium (Boice et al., 2008). Ce n'est que très récemment qu'une cohorte de millers d'uranium français, employés depuis 1955, a pu être assemblée et son profil de mortalité étudié (Bouet et al., 2018). Sur l'ensemble de cette cohorte de 1300 personnes, le risque de mortalité est globalement comparable à celui de la population générale. Aucun excès significatif de cancer du poumon ou du rein n'est observé, en cohérence avec la plupart des études menées dans d'autres pays (Pinkerton et al., 2004 ; Boice et al., 2008; Zablotska et al., 2013 ; Kreuzer et al., 2015a). Dans un sous-groupe de 552 travailleurs embauchés dans le secteur de la fabrication (soit, à différentes étapes de production du concentré d'uranium, qu'il s'avère difficile de dissocier rétrospectivement), des excès de mortalité par plusieurs pathologies ont été observés par comparaison avec la population générale locale mais, en raison d'un manque de données d'exposition dans cette cohorte, il n'est pas possible à ce jour d'identifier les facteurs de risque à l'origine de ces excès (Bouet et al., 2018).

\subsection{Cohorte des TRAvailleurs du CYcle de l'uranium (TRACY)}

La cohorte TRACY, incluant 12700 travailleurs du cycle de l'uranium impliqués depuis 1958 dans des étapes comme la conversion chimique de l'uranium, son enrichissement, la fabrication du combustible, la décontamination et différentes activités de recherche, a été mise en place depuis 2006 (Samson et al., 2016). L'un des objectifs de l'étude TRACY est de prendre en compte le contexte de multi-expositions, notamment radiologique et chimique, propre aux différentes étapes du cycle de l'uranium. Pour cela, un programme de développement de matrices emplois-expositions a été développé (Guseva Canu et al., 2009, 2013) et se poursuit actuellement (Laurent et al., 2016b). L'objectif final est de couvrir l'ensemble des établissements inclus dans le périmètre de TRACY par ces matrices emplois-expositions. Par ailleurs, la reconstitution des expositions internes aux radionucléides et l'estimation des doses en résultant nécessitent une saisie de l'ensemble des données de surveillance radiotoxicologique, qui n'ont pas toujours été centralisées dans des bases de données informatiques. Cette étude implique donc un retour systématique aux dossiers médicaux des travailleurs. Cette démarche est également l'occasion de récolter systématiquement des informations sur des facteurs de risque de pathologies renseignées dans les dossiers médicaux comme le tabagisme, la tension artérielle ou la glycémie, ce qui permet leur prise en compte dans des analyses statistiques (Zhivin et al., 2017). Les calculs de doses internes étant complexes et nécessitant de nombreuses données d'entrée, l'étude TRACY nécessite une collaboration étroite entre épidémiologistes, dosimétristes et biostatisticiens (Laurent et al., 2016a).

Sur la base d'un suivi épidémiologique jusqu'à la fin de l'année 2008, à l'échelle de l'ensemble de la cohorte TRACY, un déficit de mortalité a été observé par rapport à la population générale, reflétant l'effet du « travailleur sain » (Samson et al., 2016), classiquement observé dans des cohortes professionnelles (Goldberg and Luce, 2001). Une étude pilote portant sur un sous-ensemble de 2897 travailleurs de la cohorte a rapporté des associations positives entre l'exposition cumulée à des formes insolubles d'uranium, estimée via une matrice emploisexpositions, et les risques de cancer du poumon et de cancers lympho-hématopoïétiques (pour les composés uranifères issus du retraitement uniquement) ou encore de pathologies de l'appareil circulatoire. Ces résultats préliminaires sont basés sur des effectifs limités et doivent donc être interprétés avec précaution (Guseva Canu et al., 2014). A contrario, une étude similaire sur un autre sous-ensemble de la cohorte, concernant 4688 travailleurs de l'enrichissement, n'a pas rapporté d'association significative (Zhivin et al., 2016). Une étude cas-témoins nichée dans la cohorte TRACY suggère que la prise en compte des facteurs de risque cardiovasculaires classiques influe peu sur la relation entre dose interne résultant 
d'incorporations d'uranium et mortalité cardiovasculaire (Zhivin et al., 2017). À nouveau, en raison d'effectifs limités et de sources d'incertitudes non prises en compte jusqu'alors, les résultats de ces études doivent être considérés comme préliminaires et interprétés avec prudence.

Compte tenu des fluctuations de résultats observés entre sites géographiques, il s'avère nécessaire de répliquer les analyses sur l'ensemble du périmètre de l'étude TRACY, idéalement sur la base des estimations des doses internes reçues par les travailleurs. La conduite d'études conjointes internationales permettrait de donner encore davantage de précision statistique aux résultats et de limiter l'influence des fluctuations aléatoires (Laurent et al., 2016a). Des démarches visant à la préparation d'une telle étude ont été entreprises dans le cadre du projet européen Concerted Uranium Research in Europe (CURE). Ce projet a notamment abouti à la rédaction de protocoles dosimétriques et épidémiologiques harmonisés entre plusieurs pays européens (Laurent et al., 2016a). À ce jour, les trois plus larges études menées chez les travailleurs de l'uranium dans lesquelles des estimations de doses internes ont pu être utilisées, ne mettent pas en évidence d'association significative avec la mortalité par cancer pulmonaire (Dupree et al., 1995; Grellier et al., 2017; Yiin et al., 2017). L'une d'entre elles rapporte en revanche une association significative avec le myélome multiple (Yiin et al., 2017).

\subsection{Cohortes CEA-AREVA NC et EDF.}

Les cohortes CEA-AREVA NC et EDF ont été mises en place dans les années 1990 afin d'étudier le risque de cancer en lien avec l'exposition externe aux rayonnements ionisants chez les travailleurs de l'industrie électronucléaire. Ces deux cohortes ont été fusionnées en une cohorte conjointe incluant près de 60000 personnes et dont les principaux atouts sont la qualité de la reconstitution dosimétrique annuelle pour chaque travailleur sur l'ensemble de sa carrière (avec des premières embauches datant de 1950) et la qualité du suivi de la mortalité sur une durée moyenne de 25 ans (Leuraud et al., 2017). Dans cette cohorte, un fort effet du travailleur sain a été observé, avec un déficit de mortalité pour toutes causes confondues de près de $40 \%$ par rapport à la population générale. Ce déficit était de $32 \%$ pour les cancers solides. Des excès significatifs de cancer de la plèvre et de mélanome malin cutané ont été observés, sans lien toutefois avec l'exposition professionnelle aux rayonnements ionisants. Une augmentation non statistiquement significative (associée à une large incertitude) du risque de décès avec la dose cumulée de rayonnements ionisants a été observée pour les cancers solides, tandis qu'une augmentation significative du risque de décès avec la dose cumulée a été mise en évidence pour les leucémies myéloïdes (Leuraud et al., 2017). Une mise à jour du suivi de cette cohorte est en cours, le suivi précédent ayant été réalisé jusque fin 2004.

Cette cohorte a récemment été incluse dans l'étude internationale INWORKS rassemblant plus de 300000 travailleurs du nucléaire de trois pays (France, RoyaumeUni et États-Unis) (Hamra et al., 2016). L'étude INWORKS a mis en évidence une augmentation du risque de décès associé à la dose cumulée de rayonnements ionisants pour les leucémies (Leuraud et al., 2015), les cancers solides (Richardson et al.,
2015 ) et les maladies de l'appareil circulatoire (Gillies et al., 2017b). Pour les cancers, les coefficients de risque estimés dans INWORKS sont cohérents avec les coefficients de risque dérivés du suivi de la cohorte des survivants des bombardements d'Hiroshima et de Nagasaki, sur lesquels reposent les hypothèses sous-jacentes au système de radioprotection actuellement en vigueur (ICRP, 2007).

\section{Discussion}

Les études de cohortes de travailleurs du cycle électronucléaire menées par l'IRSN ont d'ores et déjà produit, ou contribué à produire dans le cadre de collaborations internationales, des connaissances scientifiques précieuses à l'évaluation des normes de radioprotection. Elles disposent d'un fort potentiel pour devenir encore plus informatives dans les années à venir.

\subsection{Principaux atouts des cohortes}

Parmi les forces principales de ces cohortes, outre leur pertinence pour étudier des questions de recherche prioritaires en radioprotection (HLEG, 2009; ICRP, 2017 ; Kreuzer et al., 2018), elles fournissent des connaissances précieuses à l'expertise en santé au travail et en santé publique (Laurier and Hill, 2013; Zerbib, 2018).

À ce jour, les résultats issus des cohortes de travailleurs du cycle électronucléaire apparaissent compatibles avec les hypothèses sous-jacentes au système de radioprotection actuel (ICRP, 2007). En se basant sur les résultats de l'étude INWORKS, on estime que moins de $2 \%$ des décès par cancer dans cette population de travailleurs seraient attribuables à l'exposition professionnelle aux rayonnements ionisants. Ce chiffre est à comparer avec les effets d'autres cancérogènes comme le tabac, responsable de $13 \%$ des décès en France métropolitaine (Bonaldi et al., 2016), l'alcool, la surcharge pondérale et les infections responsables respectivement de près de $8 \%$ (Shield et al., 2018a), $5 \%$ (Arnold et al., 2018) et $4 \%$ (Shield et al., 2018b) des cancers en France, sans oublier les rôles d'autres expositions environnementales ou professionnelles. En France, la valeur limite annuelle réglementaire d'exposition professionnelle aux rayonnements ionisants chez les travailleurs de catégorie A (excluant d'emblée les femmes enceintes, les femmes allaitant et les jeunes de 16 à 18 ans pour lesquels des réglementations spécifiques existent) est de 20 milliSieverts (Code du travail articles R.4451-1 et suivants). En pratique aujourd'hui, sauf situation accidentelle, les expositions professionnelles sont généralement beaucoup plus faibles (IRSN, 2017a).

Ensemble, ces cohortes représentent une population de plus de 90000 travailleurs, inclus ou en cours d'inclusion. Disposer de cohortes de cette taille s'avère indispensable pour mettre en évidence de faibles accroissements de risque associés à des expositions à de faibles doses de rayonnements ionisants, lorsqu'ils existent (Richardson et al., 2015). Les durées de suivi de plusieurs décennies atteintes par ces études, qui de par leur nature sont réalisées au long cours, contribuent également à les rendre de plus en plus informatives vis-à-vis des effets tardifs d'expositions chroniques, se cumulant lentement au cours du temps. 
Ces cohortes disposent de données de très bonne qualité, grâce au très bon suivi et à la grande stabilité professionnelle des populations incluses. Ceci se traduit notamment par un taux de perdus de vue de l'ordre de $1 \%$ seulement et une connaissance des causes de décès pour plus de $98 \%$ des travailleurs décédés en moyenne. La reconnaissance au niveau international de la qualité de ces données a permis l'inclusion de ces cohortes dans des analyses internationales (NRC, 1999; Cardis et al., 2005; Leuraud et al., 2011; Richardson et al., 2015), qui contribuent encore à augmenter la puissance statistique disponible et à exprimer ainsi le plein potentiel d'information des cohortes.

\subsection{Principales limites des études}

La prise en compte de certains facteurs de risque de pathologies chroniques autres que les rayonnements ionisants ne peut à ce stade être systématiquement effectuée dans chaque étude, faute de disponibilité immédiate de données sur ces facteurs. La suspicion selon laquelle ces facteurs pourraient exercer le rôle de facteurs de confusion dans la relation entre exposition professionnelle aux rayonnements ionisants et risques de pathologies demeure fortement ancrée. Ceci est en partie dû au fait que les conditions nécessaires pour qu'un facteur puisse exercer le rôle de facteur de confusion demeurent mal connues (Greenland et al., 1999). Disposer de données ad hoc sur certains facteurs peut néanmoins permettre de tester l'existence de confusion résiduelle. À ce jour, la plupart des études ayant mis en œuvre cette démarche ont confirmé les relations initialement observées (Leuraud et al., 2011 ; Drubay et al., 2015 ; Grellier et al., 2017 ; Zhivin et al., 2017).

Une limite des études de cohortes présentées ci-dessus est de ne considérer que la mortalité comme indicateur de santé d'intérêt principal. Les pathologies chroniques comme les cancers ou les maladies de l'appareil circulatoire n'entraînant pas toutes la même létalité, beaucoup de cas de pathologies incidentes ne peuvent être reflétés par des données de mortalité. L'incidence de pathologies, c'est-à-dire le diagnostic initial de nouvelles pathologies, constituerait un indicateur plus exhaustif de l'état de santé des populations. Cependant en France, s'il existe un enregistrement individuel exhaustif des causes de décès au niveau national depuis 1968, il n'en est pas de même pour les cas incidents de cancers ou de pathologies de l'appareil circulatoire. Pour les cancers, seuls 14 registres de cancers généraux existent en France métropolitaine, couvrant environ $24 \%$ de la population française sur 19 départements. Les registres des cardiopathies ischémiques ou des accidents vasculaires cérébraux sont encore plus rares.

Les inévitables erreurs de mesure sur les expositions aux rayonnements ionisants, ainsi que l'incertitude sur les estimations de dose utilisées dans les modèles dose-risque, sont encore rarement prises en compte dans les estimations de risques radio-induits. Cela peut générer, dans certains cas des biais dans les estimations de risques, une déformation des relations dose-réponse et/ou une sur- ou sous-estimation de l'incertitude associée aux estimations de risque. Cependant, des méthodologies statistiques avancées, visant notamment à prendre en compte certaines sources d'incertitudes dans l'estimation des relations dose-risque sont développées (Allodji et al., 2012; Hoffmann et al., 2017) et contribuent à améliorer encore la qualité de ces études.

Sauf cas particuliers (par exemple, le risque de cancer du poumon chez les mineurs d'uranium (Rage et al., 2018) ou de leucémie myéloïde dans la cohorte CEA-AREVA NC-EDF (Leuraud et al., 2017), les études réalisées au niveau national ne présentent pas une taille suffisante pour obtenir des résultats significatifs et pour quantifier, le cas échéant, la relation doseréponse pour chaque type de cancer. Plus les doses reçues sont faibles et plus les niveaux de doses au sein de la cohorte sont homogènes, plus ce constat prévaut (UNSCEAR, 2008). Seule la réalisation d'analyses conjointes de grande ampleur, notamment au niveau international, peut alors fournir la taille nécessaire à ce type d'analyse (Richardson et al., 2018).

Une limite majeure est que les cohortes existantes, même si elles couvrent les principales étapes du cycle du combustible nucléaire, n'incluent pas à ce jour la totalité des personnes ayant travaillé dans ce cycle. En effet, l'un des objectifs de ces études épidémiologiques étant d'étudier les risques chroniques associés à des expositions prolongées, les critères d'inclusion définis dans les cohortes visent à garantir la meilleure qualité de données et la meilleure traçabilité possibles à long terme. Ainsi, dans les études actuellement conduites à l'IRSN, seuls les travailleurs statutaires sont inclus car ils sont relativement stables dans leur emploi et leur historique de carrière peut être retracé très efficacement sur plusieurs années voire décennies. Les travailleurs non statutaires intervenant aux différentes phases du cycle du combustible, souvent employés par des entreprises extérieures et ne figurant donc pas dans les registres des employés des entreprises participantes ne sont donc pas inclus. C'est le cas en particulier de nombreux travailleurs effectuant des activités de maintenance sur les installations du cycle du combustible ou dans les centrales lors des arrêts de tranche et dont les expositions ont pu être plus importantes, bien qu'elles enregistrent également un recul (IRSN, 2017a). À ce jour, une seule étude française a essayé de considérer ces travailleurs des entreprises contractantes du nucléaire (Guerin et al., 2009). Cette étude portait sur 11500 travailleurs issus de 11 entreprises prestataires d'EDF. Néanmoins, puisqu'il était difficile d'identifier correctement les travailleurs et surtout de reconstituer leurs expositions de manière exhaustive sur l'ensemble de leur carrière, l'analyse de mortalité ne portait que sur $78 \%$ de la population incluse et l'analyse de la relation dose-risque ne portait que sur $55 \%$ de l'effectif cible.

\subsection{Perspectives}

La première perspective pour améliorer les études en cours est d'étendre le recueil de données d'exposition afin d'améliorer la caractérisation de certaines expositions radiologiques. Par exemple pour les contaminations par l'uranium, les données de surveillance radiotoxicologiques doivent idéalement être croisées avec des matrices emplois-expositions renseignant les formes physico-chimiques des composés uranifères rencontrés pour pouvoir estimer les dose internes en résultant (Zhivin et al., 2017). Un autre enjeu de cette démarche est la prise en compte de multi-expositions, par exemple, aux rayonnements ionisants mais également à d'autres agents physiques comme le bruit ou la chaleur, ou 
encore à des substances chimiques (Samson et al., 2016). Une estimation de ces multi-expositions permet leur prise en compte explicite au stade des analyses statistiques (Zhivin et al., 2016) en utilisant des méthodes adaptées, évitant notamment les problèmes de multicolinéarité, dans l'estimation des relations exposition-risque. Cette démarche, entreprise dans le cadre de l'étude TRACY, grâce à la construction progressive de matrices emplois-expositions, établissement par établissement, devra être poursuivie sur l'ensemble du périmètre de cette étude (Samson et al., 2016).

Le recueil de données complémentaires dans les dossiers individuels de la médecine du travail sur différents facteurs de risque, professionnels comme non professionnels (tabagisme, indice de masse corporelle...) doit également être poursuivi pour améliorer leur prise en compte dans les analyses statistiques. Comme expliqué ci-dessus, cette approche est progressivement appliquée à l'ensemble de la cohorte TRACY (Samson et al., 2016) et devra être menée jusqu'à son terme, afin que cette cohorte puisse exprimer tout son potentiel d'information. La conduite d'études cas-témoins nichées sur la mortalité par pathologies de l'appareil circulatoire pourrait également être envisagée au sein de la cohorte CEA-AREVA NC-EDF.

De nouvelles bases de données nationales aujourd'hui disponibles ouvrent des perspectives prometteuses pour la prise en compte de l'incidence de pathologies au sein des études de cohortes. En particulier, la base du Système National d'Information Inter-Régimes de 1'Assurance Maladie (SNIIRAM) créée en 1999 est potentiellement d'intérêt majeur à cette fin (Goldberg et al., 2016). L'IRSN a obtenu un décret du Conseil d'État pour certaines de ses études épidémiologiques réalisées dans le domaine médical, permettant d'accéder aux données de la base du SNIIRAM et ainsi d'obtenir des informations de santé complémentaires afin de pouvoir travailler avec des données d'incidence. Si ces premiers croisements sont informatifs, des demandes d'autorisation seront faites afin d'étendre l'utilisation du SNIIRAM aux études épidémiologiques des travailleurs du cycle électronucléaire. La faisabilité de reconstitution d'historiques d'expositions médicales aux rayonnements ionisants via le SNIIRAM pourrait également être envisagée, sous réserve d'obtention des autorisations nécessaires. En parallèle, l'exploitabilité de systèmes d'enregistrement des pathologies incidentes propres aux entreprises a pu être étudiée (Chevalier et al., 1996; Roué et al., 2015). Ces systèmes présentant certaines limites, ils pourront être utilisés comme sources complémentaires d'informations.

Une perspective majeure des études à venir chez les travailleurs du nucléaire est la poursuite de développements méthodologiques afin d'intégrer les sources d'incertitudes les plus importantes dans l'estimation des modèles dose-risque. Ceci permettra d'évaluer l'impact de ces incertitudes sur l'estimation des risques de survenue de pathologies radioinduites et soit de conforter, soit d'affiner les estimations actuelles de risque. Ces perspectives font nécessairement appel à d'étroites collaborations multidisciplinaires entre dosimétristes, biostatisticiens et épidémiologistes.

L'extension du suivi des études est essentielle et constitue également une perspective majeure puisque les cohortes, au fur et à mesure de leur suivi, sont de plus en plus informatives sur l'expression des risques chroniques associés à des expositions prolongées (Leuraud et al., 2015 ; Richardson et al., 2015). À court terme, trois perspectives majeures sont les extensions des suivis des cohortes CEA-AREVA NC-EDF (dont le suivi actuel s'arrête en 2004) (Leuraud et al., 2017), TRACY (dont le suivi actuel s'arrête en 2008) (Samson et al., 2016) et de la cohorte des mineurs d'uranium (dont le suivi actuel s'arrête en 2007) (Rage et al., 2018). Les analyses internationales ne dérogeant pas à cette règle, des mises à jour des analyses internationales précédentes (NRC, 1999; Hamra et al., 2016) sont envisagées et la participation des cohortes de l'IRSN est sollicitée dans plusieurs d'entre elles. De telles études sont indispensables pour que les cohortes puissent exprimer tout leur potentiel d'information.

Depuis le début des années 2000, chaque exploitant nucléaire en France est réglementairement tenu de transmettre au système SISERI (Biau, 2011), géré par l'IRSN (IRSN, 2018), l'ensemble des enregistrements des doses externes reçues par ses travailleurs et depuis 2009, des données relatives à la contamination interne sont également enregistrées dans SISERI. Il apparaît donc maintenant possible de suivre les travailleurs des entreprises contractantes avec une plus grande fiabilité que par le passé. L'inclusion de ces travailleurs prestataires dans les études épidémiologiques de l'IRSN est donc envisagée.

Enfin, les cohortes de travailleurs du nucléaire doivent tendre à intégrer le concept d'exposome (Wild, 2005) de plus en plus plébiscité dans la recherche en santé publique. L'exposome, pendant du génome pour ce qui concerne les expositions environnementales (incluant notamment les expositions associées aux comportements de consommation), comprend dans sa définition large l'ensemble des expositions physiques, chimiques et biologiques mais aussi psychosociales, en intégrant leurs variations temporelles. Les « régulations du milieu intérieur »en réponse à ces expositions, comme d'ailleurs au stress psychosocial, sont également intégrées dans cette notion (Barouki, 2014).

Plusieurs approches, non exclusives, peuvent permettre d'intégrer au moins en partie l'exposome dans les cohortes rétrospectives de travailleurs du nucléaire. La construction de matrices emplois-expositions, déjà évoquée ci-dessus, vise précisément à documenter et à prendre en compte le contexte de multi-expositions des travailleurs, même si elle se cantonne dans ce cas à tenir compte de leurs expositions professionnelles. Des analyses récemment menées dans la cohorte CEAAREVA NC-EDF ont également permis de prendre en compte l'exposition environnementale des travailleurs aux rayonnements ionisants (Fournier et al., 2018). D'autres exemples de prise en compte d'expositions environnementales dans des cohortes professionnelles existent, parmi lesquelles l'étude de la relation entre exposition à la pollution de l'air et mortalité au sein de la cohorte GAZEL (Bentayeb et al., 2015). Il serait donc techniquement envisageable d'appliquer cette démarche à d'autres cohortes professionnelles et à d'autres expositions environnementales.

Enfin, un dernier axe envisageable, sous réserve d'obtention des autorisations nécessaires (en réponse à une question posée dans un éditorial de Radioprotection [Grosche, 2017]), est la réalisation d'études en épidémiologie moléculaire, permettant l'intégration de données biologiques reflétant les «régulations du milieu intérieur»au sein de cohortes prospectives faisant appel à des volontaires (Gueguen et al., 2017). 
Les cohortes prospectives se prêtent en effet à la réalisation de mesures répétées indispensables pour intégrer les variations temporelles de biomarqueurs, en amont de l'apparition de pathologies, ce qui permet d'éviter tout biais dit de «causalité inverse» (Wild, 2005). Même si aucun biomarqueur idéal d'effet des radiations n'a encore pu être identifié à ce jour (Hall et al., 2017) (ce qui ne permet pas de préjuger des évolutions futures dans ce domaine), cette démarche serait pertinente pour la prise en compte de l'exposome. Des intérêts majeurs pourraient être par exemple l'identification de biomarqueurs d'expositions à d'autres facteurs de risque suspectés de jouer le rôle de facteur de confusion, la mise en évidence de mécanismes d'action des radiations aux faibles doses, voire l'identification de populations présentant une radiosusceptibilité particulière. De par la nature complexe de l'exposome et la quantité de données nécessaire pour l'aborder, sa prise en compte implique des développements méthodologiques et des collaborations pluridisciplinaires étroites entre différents partenaires (Laurent et al., 2016a).

\section{Financements}

Institut de Radioprotection et de Sûreté Nucléaire Commission Européenne (projet ALPHARISK)

Commission Européenne et réseau d'excellence DoReMi (Projet CURE)

Électricité De France (accord bilatéral de cofinancement IRSN/EDF)

AREVA (accord bilatéral de cofinancement IRSN/ AREVA)

Les co-financeurs n'ont pas participé à la conception des protocoles d'études, l'analyse des données, ni à l'interprétation des résultats, qui relèvent de la seule responsabilité de l'IRSN.

Accord CNIL : numéro DR-2012-611

Remerciements. Les auteurs remercient le Dr Bernard le Guen (EDF) pour ses commentaires sur cet article. Ils remercient également l'ensemble des personnes qui contribuent à la réalisation de ces études en permettant l'accès aux sources d'informations administratives, dosimétriques et médicales et en apportant leur connaissance de l'histoire des entreprises et des conditions de travail : les responsables des entreprises, les médecins du travail, infirmiers, ergonomes et autres membres des services de santé au travail, les personnes compétentes en radioprotection, les représentants du personnel et les travailleurs et ex-travailleurs des sites. Ils remercient enfin leurs collègues qui, avant eux, ont travaillé au développement de ces études.

\section{Références}

Allodji RS et al. 2012. The performance of functional methods for correcting non-Gaussian measurement error within Poisson regression: corrected excess risk of lung cancer mortality in relation to radon exposure among French uranium miners. Stat. Med. 31: 4428-4443.

Arnold M et al. 2018. Cancers in France in 2015 attributable to high body mass index. Cancer Epidemiol. 52: 15-19.

Azizova TV, Haylock RG, Moseeva MB, Bannikova MV, Grigoryeva ES. 2014. Cerebrovascular diseases incidence and mortality in an extended mayak worker cohort 1948-1982. Radiat. Res. 182: 529-544.

Barouki R. 2014. Environnement et santé: la combinatoire des expositions. Quest. Santé Publique. Inst. Rech. Santé Publique 26: $1-4$.

Bentayeb M et al. 2015. Association between long-term exposure to air pollution and mortality in France: A 25-year follow-up study. Environ. Int. 85: 5-14.

Biau A. 2011. Exploitation des données dosimétriques, les dossiers médicaux, SISERI. Radioprotection 46: S205-S211.

Boice JD, Jr., Cohen SS, Mumma MT, Chadda B, Blot WJ. 2008. A cohort study of uranium millers and miners of Grants, New Mexico, 1979-2005. J. Radiol. Prot. 28: 303-325.

Boice JD, Jr., et al. 2014. Mortality among mound workers exposed to polonium-210 and other sources of radiation, 1944-1979. Radiat. Res. 181: 208-228.

Bonaldi C et al. 2016. Les décès attribuables au tabagisme en France. Dernières estimations et tendance, années 2000 à 2013. Bull. Epidémiol. Hebd. 30-31: 528-540.

Bouet S, Samson E, Jovanovic I, Laurier D, Laurent O. 2018. First mortality analysis in the French cohort of uranium millers (FMillers), period 1968-2013. Int. Arch. Occup. Environ. Health 91: 23-33.

Bourguignon M, Bérard P, Bertho JM, Farah J, Mercat C. 2017. Radioprotection: quel avenir? Radioprotection 52: 13-20.

Cardis E et al. 2005. Risk of cancer after low doses of ionising radiation: retrospective cohort study in 15 countries. BMJ 331: 77.

Chevalier A et al. 1996. Cancer incidence among active male workers at Électricite de France-Gaz de France. Rev. Epidemiol. Santé Publique 44: 25-36.

Daniels RD et al. 2017. Examining temporal effects on cancer risk in the international nuclear workers' study. J. Int. Cancer 140: 12601269.

Drubay D, Ancelet S, Acker A, Kreuzer M, Laurier D, Rage E. 2014. Kidney cancer mortality and ionizing radiation among French and German uranium miners. Radiat. Environ. Biophys. 53: 505-513.

Drubay D, Caer-Lorho S, Laroche P, Laurier D, Rage E. 2015. Mortality from circulatory system diseases among French uranium miners: A nested case-control study. Radiat. Res. 183: 550-562.

Dupree EA, Watkins JP, Ingle JN, Wallace PW, West CM, Tankersley WG. 1995. Uranium dust exposure and lung cancer risk in four uranium processing operations. Epidemiology 6: 370-375.

Fournier L, Clero E, Samson E, Caer-Lorho S, Laurier D, Leuraud K. 2018. Impact of considering non-occupational radiation exposure on the association between occupational dose and solid cancer among French nuclear workers. Occup. Environ. Med. 75: 199204.

Gilbert ES et al. 2013. Lung cancer risks from plutonium: an updated analysis of data from the Mayak worker cohort. Radiat. Res. 179: 332-342.

Gillies M et al. 2017a. Lung cancer risk from plutonium: A pooled analysis of the Mayak and Sellafield worker cohorts. Radiat. Res. 188: 645-660.

Gillies M et al. 2017b. Mortality from circulatory diseases and other non-cancer outcomes among nuclear workers in France, the United Kingdom and the United States (INWORKS). Radiat. Res. 188: 276-290.

Goldberg M, Luce D. 2001. Selection effects in epidemiological cohorts: nature, causes and consequences. Rev. Epidemiol. Santé Publique 49: 477-492.

Goldberg M et al. 2016. The opening of the French national health database: Opportunities and difficulties. The experience of the 
Gazel and Constances cohorts. Rev. Epidemiol. Santé Publique 64: 313-320.

Grant EJ et al. 2017. Solid cancer incidence among the life span study of atomic bomb survivors: 1958-2009. Radiat. Res. 187: 513-537.

Greenland S, Pearl J, Robins JM. 1999. Causal diagrams for epidemiologic research. Epidemiology 10: 37-48.

Grellier J et al. 2017. Risk of lung cancer mortality in nuclear workers from internal exposure to alpha particle-emitting radionuclides. Epidemiology 28: 675-684.

Grosche B. 2017. Editorial comment on: Mortality in the French cohort of nuclear workers. Radioprotection 52: 211-212.

Gueguen Y et al. 2017. Biomarkers for uranium risk assessment for the development of the cure (Concerted Uranium Research in Europe) molecular epidemiological protocol. Radiat. Res. 187: $107-127$.

Guerin S et al. 2009. Cancer mortality among French nuclear contract workers. Am. J. Ind. Med. 52: 916-925.

Guseva Canu I et al. 2009. Comparative assessing for radiological, chemical, and physical exposures at the French uranium conversion plant: Is uranium the only stressor? Int. J. Hyg. Environ. Health 212: 398-413.

Guseva Canu I, Faust S, Knieczak E, Carles M, Samson E, Laurier D. 2013. Estimating historic exposures at the European gaseous diffusion plants. Int. J. Hyg. Environ. Health 216: 499-507.

Guseva Canu I et al. 2014. Effects of chronic uranium internal exposure on mortality: results of a pilot study among French nuclear workers. Rev. Epidemiol. Santé Publique 62: 339-350.

Hall J et al. 2017. Ionizing radiation biomarkers in epidemiological studies - An update. Mutat. Res. 771: 59-84.

Hamra GB et al. 2016. Cohort Profile: The International Nuclear Workers Study (INWORKS). Int. J. Epidemiol. 45: 693-699.

HLEG. 2009. HLEG report on European low dose risk research. EUR 23884. Luxembourg, UK: Office for Official Publications of the European Communities.

Hoffmann S, Rage E, Laurier D, Laroche P, Guihenneuc C, Ancelet S. 2017. Accounting for Berkson and classical measurement error in radon exposure using a Bayesian structural approach in the analysis of lung cancer mortality in the French cohort of uranium miners. Radiat. Res. 187: 196-209.

ICRP Publication 103. 2007. The 2007 Recommendations of the International Commission on Radiological Protection. Ann. ICRP 37.

ICRP. 2017. Areas of Research to Support the System of Radiological Protection. ICRP ref 4832-9526-9446. Available from http://www. icrp.org/docs/ICRP\%20Research\%20Priorities\%202017.pdf.

IRSN. 2017a. La radioprotection des travailleurs Exposition professionnelle aux rayonnements ionisants en France: bilan 2016. Fontenay-aux-Roses: IRSN. Disponible sur http://www. irsn.fr/FR/expertise/rapports_expertise/Documents/radioprotec tion/IRSN_Rapport-Exposition-travailleurs-2016.pdf.

IRSN. 2017b. Suivi épidémiologique des travailleurs du cycle électronucléaire en France : bilan des études de l'IRSN. Rapport $n^{o}$ PSE-SANTE/SESANE/2017-001. Disponible sur http://www. irsn.fr/FR/expertise/rapports_expertise/Documents/radioprotec tion/IRSN-Rapport-Suivi-epidemio-travailleurs-nucle aire_201712.pdf. Fontenay-aux-Roses.

IRSN. 2018. Système d'information de la surveillance de l'exposition aux rayonnements ionisants. Disponible sur http://siseri.irsn.fr/. Consulté le 8 mars 2018 (Type of Medium).

Kreuzer M, Grosche B, Schnelzer M, Tschense A, Dufey F, Walsh L. 2010. Radon and risk of death from cancer and cardiovascular diseases in the German uranium miners cohort study: follow-up 1946-2003. Radiat. Environ. Biophys. 49: 177-185.
Kreuzer M et al. 2015a. Mortality from internal and external radiation exposure in a cohort of male German uranium millers, 1946-2008. Int. Arch. Occup. Environ. Health 88: 431-441.

Kreuzer M, Fenske N, Schnelzer M, Walsh L. 2015b. Lung cancer risk at low radon exposure rates in German uranium miners. $\mathrm{Br}$. $J$. Cancer 113: 1367-1369.

Kreuzer M et al. 2018. Multidisciplinary European Low Dose Initiative (MELODI): strategic research agenda for low dose radiation risk research. Radiat. Environ. Biophys. 57: 5.

Laurent $\mathrm{O}$ et al. 2016a. Concerted uranium research in Europe (CURE): toward a collaborative project integrating dosimetry, epidemiology and radiobiology to study the effects of occupational uranium exposure. J. Radiol. Prot. 36: 319-345.

Laurent O, Villeneuve S, Samson E, Laroche P, Serond AP, Laurier D. 2016b. New job exposure matrices for epidemiological studies in French uranium workers. Radiation Protection Week 19-23, Oxford (UK).

Laurier D, Hill C. 2013. Risque de cancer lié aux radiations ionisantes. Rev. Prat. 63: 1126-1132.

Laurier D et al. 2004. An update of cancer mortality among the French cohort of uranium miners: extended follow-up and new source of data for causes of death. Eur. J. Epidemiol. 19: 139-146.

Laurier D et al. 2012. DoReMi workshop on multidisciplinary approaches to evaluating cancer risks associated with low-dose internal contamination. Radioprotection 47: 119-148.

Leuraud $\mathrm{K}$ et al. 2007. Lung cancer risk associated to exposure to radon and smoking in a case-control study of French uranium miners. Health Phys. 92: 371-378.

Leuraud K et al. 2011. Radon, smoking and lung cancer risk: results of a joint analysis of three European case-control studies among uranium miners. Radiat. Res. 176: 375-387.

Leuraud $\mathrm{K}$ et al. 2015. Ionising radiation and risk of death from leukaemia and lymphoma in radiation-monitored workers (INWORKS): an international cohort study. Lancet Haematol. 2: e276-281.

Leuraud K, Fournier L, Samson E, Caër-Lorho S, Laurier D. 2017. Mortality in the French cohort of nuclear workers. Radioprotection 52: 199-210.

McLean AR et al. 2017. A restatement of the natural science evidence base concerning the health effects of low-level ionizing radiation. Proceedings. Biol. Sci. 284.

NRC. 1999. National Research Council, Committee on Health Risks of Exposure to Radon. Health effects of exposure to radon (BEIR VI). Washington, DC: National Academy Press.

Ozasa K et al. 2012. Studies of the mortality of atomic bomb survivors, Report 14, 1950-2003: an overview of cancer and noncancer diseases. Radiat. Res. 177: 229-243.

Pinkerton LE, Bloom TF, Hein MJ, Ward EM. 2004. Mortality among a cohort of uranium mill workers: an update. Occup. Environ. Med. 61: 57-64.

Rage E, Caer-Lorho S, Laurier D. 2018. Low radon exposure and mortality among Jouac uranium miners: an update of the French cohort (1946-2007). J. Radiol. Prot. 38: 92-108

Repussard J. 2017. Low dose effects research in Europe: eight years of evolution towards new paradigms. Radioprotection 52: 251258.

Richardson DB et al. 2015. Risk of cancer from occupational exposure to ionising radiation: retrospective cohort study of workers in France, the United Kingdom, and the United States (INWORKS). BMJ 351: h5359.

Richardson DB et al. 2018. Site-specific solid cancer mortality after exposure to ionizing radiation: A cohort study of workers (INWORKS). Epidemiology 29: 31-40. 
Roué T et al. 2015. Croisement des données d'incidence de cancer issues d'un service de santé au travail avec celles issues de registres de cancers. Arch. Mal. Prof. Environ. 76: 231-236.

Samson E et al. 2016. Cancer and non-cancer mortality among French uranium cycle workers: the TRACY cohort. BMJ Open 6: e010316.

Shield KD et al. 2018a. New cancer cases in France in 2015 attributable to different levels of alcohol consumption. Addiction . DOI: $10.1111 /$ add.14009.

Shield KD et al. 2018b. New cancer cases in France in 2015 attributable to infectious agents: a systematic review and metaanalysis. Eur. J. Epidemiol. 33(3): 263-274.

UNSCEAR. 2008. UNSCEAR 2006 Report to the General Assembly with Scientific Annexes, Effects of Ionizing Radiation. Vol. 1: Report and Annexes A and B. New York: United Nations.

UNSCEAR. 2015. UNSCEAR 2012 Report to the General Assembly with Scientific Annexes, Sources and Effects of Ionizing Radiation. Vol. 2: Scientific Annex A "Attributing health effects to ionizing radiation exposure and inferring risks". New York: United Nations.
Wild CP. 2005. Complementing the genome with an "exposome": the outstanding challenge of environmental exposure measurement in molecular epidemiology. Cancer Epidemiol. Biomark. Prev. 14: $1847-1850$.

Yiin JH et al. 2017. Mortality in a combined cohort of uranium enrichment workers. Am. J. Ind. Med. 60: 96-108.

Zablotska LB, Lane RS, Frost SE. 2013. Mortality (1950-1999) and cancer incidence (1969-1999) of workers in the Port Hope cohort study exposed to a unique combination of radium, uranium and gamma-ray doses. BMJ Open 3: e002159.

Zerbib JC. 2018. Les maladies professionnelles radioinduites reconnues en France bilan et évolutions depuis 1956. Radioprotection 53: 51-60.

Zhivin S et al. 2016. Mortality (1968-2008) in a French cohort of uranium enrichment workers potentially exposed to rapidly soluble uranium compounds. Occup. Environ. Med. 73: $167-174$.

Zhivin S et al. 2017. Circulatory disease in French nuclear fuel cycle workers chronically exposed to uranium: a nested case-control study. Occup. Environ. Med. 75(4): 270-276.

Citation de l'article : Samson E, Leuraud K, Rage E, Caër-Lorho S, Ancelet S, Cléro E, Bouet S, Hoffmann S, Fournier L, Belloni M, Jovanovic I, Bah T, Davesne E, Blanchardon E, Challeton-de Vathaire C, Laurier D, Laurent O. 2018. Bilan de la surveillance épidémiologique des travailleurs du cycle électronucléaire en France. Radioprotection 53(3): 175-184 